\title{
Quelques
}

opportunités et

défis conceptuels,

fonctionnels et

politiques $d u$

fédéralisme

\section{Hugo Cyr}

\section{Introduction}

Le fédéralisme, par définition, n'est pas une chose simple. Mais les choses simples ne sont pas nécessairement les plus belles, les plus efficaces ou les meilleures. L'État-Nation souverain paraît peut-être bien simple, mais il n'est pas pour autant le meilleur agencement politique que l'on puisse imaginer en toute circonstance ${ }^{1}$. En fait, les différentes formes de fédéralisme visent particulièrement à dépasser les solutions parfois trop simplistes que le modèle de l'ÉtatNation souverain prétend offrir. Toutefois, c'est trop souvent en référence à ce dernier modèle que le fédéralisme se conçoit.

Une première série de défis qui attendent la théorie du fédéralisme relève donc du développement d'un appareillage conceptuel qui saura acquérir son autonomie à l'égard des vieilles catégories de la souveraineté (I). Une seconde série de défis découle de la première : le démembrement de la souveraineté nous invite à repenser l'attribution des diverses fonctions étatiques entre de multiples institutions et les problèmes fonctionnels qu'une telle division peut occasionner (II). Cette deuxième série appelle, à son tour, une troisième série de défis du fait que si l'efficacité et la fonctionnalité sont outils de la politique, ils ne sont pas la politique. Ainsi, cette troisième série de défis correspond aux enjeux associés à l'élaboration d'arrangements institutionnels qui chercheront à assurer un maximum de respect des diverses allégeances communautaires superposées tout en sachant se justifier à l'égard des attentes relatives à l'efficacité instrumentale abstraite de l'État moderne (III). En bref, la troisième série de défis identifiée fait le lien entre la première et la deuxième dans la mesure où il est question de trouver la forme politique adéquate qui saura concilier l'efficacité gouvernementale et le respect des multiples communautés politiques.

Ce bref article vise à présenter ces trois séries de défis tout en proposant quelques pistes théoriques et pratiques en vue de les relever. Notons toutefois que ces défis que posent les divers enchevêtrements d'allégeances constituant le tissu de légitimité des organes politiques au sein d'une Fédération - tissu que ces organes contribuent à produire et sur lequel ils reposent - sont autant d'opportunités d'élaborer un espace où le vivre-ensemble est fondé sur un meilleur respect mutuel. En ce sens, ces défis ne devraient pas être perçus comme des motifs de s'éloigner des formes fédérales, bien au contraire! Il s'agit plutôt de possibilités créatrices qui s'ouvrent afin de mieux adapter nos institutions à nos besoins concrets. 


\section{Quelques défis conceptuels du fédéralisme}

«La souveraineté est une, indivisible, inaliénable et imprescriptible » nous enseignait la Constitution française du 3 septembre 1791. Or, le premier défi pour la théorie du fédéralisme est de s'émanciper du carcan conceptuel imposé par la dominance de ce concept de souveraineté dans les discours politiques et juridiques occidentaux. Cette dominance fait en sorte que trop souvent nous nous obligeons à penser le fédéralisme à travers les contraintes conceptuelles qu'impose le modèle de l'État-Nation. C'est notamment le cas lorsque nous nous demandons où repose la souveraineté au Canada depuis que ce dernier a acquis son indépendance du Royaume-Uni : se trouve-t-elle dans la Couronne du Chef du Canada? Dans l'État fédéral? Dans la Couronne du Chef des provinces? Dans les provinces? Dans une combinaison de ces deux paliers gouvernementaux? Si la souveraineté repose dans une telle combinaison, quand et comment s'exerce-t-elle concrètement, dans la mesure où les pouvoirs étatiques ont été distribués de manière exclusive à l'un et à l'autre des paliers gouvernementaux? L'idée même de partage de la souveraineté n'est-elle pas profondément antinomique? Bref, comment pouvons-nous encore donner un sens à une souveraineté désunie, divisée et aliénée? Si la souveraineté reposedanslePeupledela Fédération canadienne, de quoi est composé celui-ci : d'individus? De nations? De communautés provinciales et territoriales? Remarquons en passant qu'un questionnement similaire est au cœur des débats quant à l'avenir de l'Union européenne. Alors que certains voient en l'Europe une Fédération en devenir - si elle n'en est pas déjà une -, d'autres estiment qu'elle devrait à tout prix se développer en respectant la sacro-sainte " souveraineté nationale ». Les tenants de cette dernière position sont allergiques à l'idée que les institutions européennes puissent obtenir une complète autonomie décisionnelle de jure des pays membres sur quelque domaine réservé.

Pour s'émanciper du carcan conceptuel qu'impose l'idée de souveraineté dans l'appréhension de différentes formes de réalités juridico-politiques, certains nient totalement son utilité en avançant, notamment, que cette notion est bien peu adaptée à une description réaliste des faits politiques contemporains auxquels elle prétend offrir un cadre analytique. Les monarchies absolutistes ne représentent plus le modèle dominant d'organisation politique. Aussi, parler de l'existence d'un pouvoir suprême unique dans le cadre d'un État où la capacité d'exercice des pouvoirs est divisée fonctionnellement et institutionnellement entre diverses branches (législative, exécutive, judiciaire) semble relever d'un exercice futile de scolastique. La distinction entre l'existence d'un pouvoir et la capacité de son exercice paraît à plusieurs bien factice. D'autres diront que pour compléter le processus de décolonisation il ne suffit pas de remplacer à la tête des gouvernements des dirigeants locaux plutôt que des colons, mais qu'il faut aussi se libérer l'esprit de concepts politiques empruntés au pouvoir colonial - tels que ceux de souveraineté et d'ÉtatNation - qui ne collent pas nécessairement aux réalités plurielles et marquées par la profonde diversité qu'est celle des peuples décolonisés. D'autres encore soulignent qu'à l'époque où nous vivons, aucune entité politique ne peut réellement se targuer d'omnipotence. La mondialisation des marchés, l'interdépendance des États et la pénétration des règles du droit international dans ce qui formait autrefois des sphères locales exclusives font en sorte qu'aucun État ne peut prétendre fonctionner sans voir au moins quelques-unes de ses décisions dictées non seulement par des forces externes, mais aussi par des normes auxquelles il n'a pas nécessairement consenti ${ }^{2}$. L'État moderne n'est ni de facto, ni de jure entièrement indépendant des autres.

Nous entendons bien ces critiques du concept de souveraineté - et de la conception de l'État-Nation qui est un de ses produits dérivés -, mais nous allons ici restreindre notre propos aux contraintes indues qu'impose ce cadre conceptuel pour comprendre le phénomène fédératif.

Tout d'abord, la critique du monisme qui sous-tend l'idée de souveraineté prend une très grande importance dans un contexte fédéral. Les pouvoirs souverains étant absolus, ils ne 
peuvent être divisés sans quoi l'acte même de division imposerait une limite à ces pouvoirs et nierait donc le caractère souverain de ceux-ci. Or, le fédéralisme, à sa base même, repose sur l'idée qu'il est possible de diviser l'ensemble des pouvoirs étatiques entre divers paliers étatiques coordonnés où aucun palier n'est un délégué de l'autre.

$\begin{array}{ccc}\text { Afin d'accommoder l'existence } & \mathrm{du} \\ \text { fédéralisme, certains chercheront } & \text { la }\end{array}$
souveraineté dans le constituant ayant mis en place la constitution fédérale. Outre le fait que cette hypothèse d'une souveraineté reposant sur le constituant originaire cadre fort mal avec la situation des Fédérations canadienne et australienne où le constituant originaire n'a plus aucun pouvoir juridique relatif à elles - ni le Canada, ni l'Australie ne sont aujourd'hui des colonies liées par les lois britanniques -, cette hypothèse ignore aussi comment l'acte fédératif même a pu transformer, voire créer des agents pouvant se réclamer de la capacité d'entité constituante. Par exemple, si l'Acte d'Amérique $d u$ Nord britannique de 1867 - aujourd'hui nommé Loi constitutionnelle de $1867^{3}$ - a donné lieu à la création de la Fédération canadienne, il a aussi permis la création des provinces autonomes de l'Ontario et de Québec que l'on connaît aujourd'hui à partir de ce qui était, depuis 1841, la Province of Canada. La Province of Quebec avait bel et bien existé de 1774 à 1791, mais elle ne couvrait environ qu'un tiers de la superficie du Québec actuel, la partie restante du territoire contemporain, ce qui était alors la Terre de Rupert, étant sous la gouverne de la Compagnie de la Baie d'Hudson. Si le territoire originel de la Province of Quebec ne couvrait qu'un tiers de ce qu'est le territoire québécois contemporain, il couvrait par ailleurs un ensemble de territoires qui en sont maintenant exclus, soit le Labrador et une partie de ce qui constitue maintenant six États américains. Autrement dit, le territoire contemporain du Québec est relativement différent de celui de la vieille Province of Quebec en ce qu'il a gagné un tiers de territoire d'un côté et en a perdu à peu près l'équivalent de l'autre. Même dans le Constitution Act of 1791 qui s'appliquera jusqu'en 1841, les Province of Upper Canada et de Lower Canada n'auront pas la même étendue territoriale que connaissent l'Ontario et le Québec actuels. En bref, bien que les entités politiques que sont aujourd'hui les provinces de l'Ontario et de Québec soient des créations de l'acte fédératif de 1867 , ces mêmes créations réclament aujourd'hui les pouvoirs d'agents constituants.

Certains diront qu'en effet, il ne faut pas chercher la souveraineté dans le pouvoir constituant originaire, mais plutôt dans le pouvoir constituant dérivé ou un nouveau pouvoir constituant originaire latent (c'est-àdire un pouvoir constituant qui ne s'est pas encore fait entendre : le peuple). Or, ces deux dernières suggestions posent évidemment d'autres difficultés.

Premièrement, dans plusieurs Fédérations, il n'existe pas une modalité unique de modification de la constitution, mais plutôt divers processus de modification constitutionnelle. Chaque processus d'amendement commande ses propres forces constituantes dérivées. Par exemple, certaines modifications constitutionnelles ne sont possibles au Canada qu'avec l'approbation unanime des dix provinces et de l'État fédéral. Pour d'autres, l'appui de sept provinces représentant $50 \%$ de la population canadienne et de l'État fédéral suffira. Et nous ne parlons pas des cas où une province seule peut modifier sa constitution ou encore modifier une partie de la Constitution canadienne avec le simple accord de l'État fédéral. Ces diverses formules reflètent une variété d'amalgames représentant des pouvoirs constituants dérivés différents les uns des autres; la souveraineté une et unique ne saurait se trouver dans cet assortiment d'acteurs collectifs.

Deuxièmement, l'hypothèse selon laquelle il pourrait y avoir une population dans laquelle résiderait un nouveau pouvoir constituant originel pouvant remplacer le Parlement impérial britannique ne tient pas compte du fait même que c'est de Fédérations dont il est question ici. Si l'État-Nation peut compter sur une communauté souveraine pour lui donner sa légitimité et si la confédération n’a de légitimité que dans la mesure où les pouvoirs délégués au centre par les États confédérés le sont en conformité à la volonté des «peuples » de ces 
États, la légitimité de la Fédération repose dans le «peuple » des États fédérés tout autant que dans le "peuple » de l'État fédéral. Autrement formulé, l'État fédéral et les États fédérés s'appuient sur la légitimité populaire, mais cette légitimité provient de corps politiques distincts pouvant être composés en bonne partie des mêmes individus. Ainsi, la théorie fédérale non seulement est prête à accommoder les allégeances politiques multiples, mais elle se justifie précisément par la possibilité de cellesci. Ce qui explique pourquoi l'acte de sécession d'un État fédéré ne correspond pas simplement à une perte de territoire pour l'État fédéral; cela correspond aussi à une amputation d'une partie de son corps politique. Mais puisque le peuple de l'État fédéral n'est pas plus légitime que le peuple de l'État fédéré, aucun des deux peuples ne peut légitimement imposer de manière unilatérale sa décision en cette matière. Ainsi, dans une Fédération, il ne peut y avoir une communauté unique en qui reposerait la souveraineté; le concept de Fédération exige que chacun des paliers possède une légitimité populaire qui lui soit propre et ainsi chacun des paliers représente une fraction des forces constituantes originaires latentes ${ }^{4}$.

Après celui de l'émancipation, le deuxième défi conceptuel que pose le fédéralisme est donc celui de la reconstruction. Plusieurs éléments sont alors à prendre en considération. Il s'agira, par exemple, d'imaginer les pouvoirs de l'État non plus selon le modèle moniste, mais plutôt selon un modèle qui reconnaît à la personne la possibilité de se vouer aux diverses communautés politiques auxquelles elle appartient simultanément. En effet, ce modèle doit permettre à la personne de faire partie de plus d'une communauté politique légitime à la fois, communautés qui peuvent se superposer sans nécessairement compter exactement les mêmes membres. L'on doit donc pouvoir concevoir l'État sans qu'il lui soit nécessaire, même en principe, d'être capable de gouverner tout aspect de la vie des personnes qui le composent. Contrairement à l'image traditionnelle de l'État n'ayant pour seules limites que les forces externes et ses décisions de s'autolimiter (incluant des règles constitutionnelles qu'il peut changer), il faut aussi accepter de penser un État aux pouvoirs limités par des règles constitutives dont il ne possède pas à lui seul la capacité de les modifier et ce, sans toutefois être subordonné à un autre pouvoir.

Aussi, dans cette reconstruction, devronsnous prendre en considération la prémisse suivante : puisque les États fédérés et l'État fédéral sont chacun responsables de différents aspects du bien-être des membres de leur communauté et que souvent ils ne peuvent assumer cette responsabilité sans des efforts concertés, l'État ne peut plus agir à sa guise dans le but d'atteindre les objectifs qu'il se donne. L'État ne doit plus être conçu comme une puissance publique s'imposant à tous à l'intérieur d'un territoire donné, mais en contexte fédéral, il doit entretenir des liens horizontaux de collaboration et de coopération avec d'autres puissances publiques légitimes sur son territoire. L'État dans la Fédération perd donc non seulement son monopole sur la violence, mais aussi son monopole sur l'usage de la puissance publique en général. Cela n'est pas une mince affaire que de repenser l'État en l'absence de ces monopoles.

Si on pousse encore un peu plus loin, on verra que le fédéralisme dit « personnel » remet encore plus en question nos conceptions de l'État en éloignant la nécessité du contrôle effectif d'un territoire ${ }^{5}$. En effet, le « fédéralisme personnel " ne repose pas sur un partage des compétences étatiques en fonction de divisions territoriales, mais reconnaît plutôt des ordres juridiques distincts applicables aux uns et aux autres en fonction de leurs caractéristiques personnelles (choisies ou non). Ces caractéristiques personnelles sont en fait les marqueurs d'appartenance à l'une ou l'autre des communautés politiques pertinentes aux fins de la fédéralisation. Ce fédéralisme personnel peut porter sur des sujets plutôt circonscrits par exemple, l'appartenance autodéclarée à une communauté linguistique pourra déterminer si une personne et ses enfants seront sous la gouverne d'un système scolaire linguistique $\mathrm{A}$ ou B - ou alors l'ordre personnel peut avoir une portée très large - par exemple, l'objet d'une fédéralisation peut être l'ensemble du droit de 
la famille. Dans un contexte où ce qui importe est la caractéristique personnelle des membres d'une communauté et non leur localisation, on peut sans doute même s'imaginer une fédéralisation qui ne s'attache pas aux sacrosaintes frontières physiques qui ont longtemps été si jalousement gardées par l’État.

Nous n'élaborerons pas ici une théorie de l'État qui prend en compte cette nouvelle donne, mais nous dirons simplement que cette nouvelle conceptualisation permettra sans doute de trouver des solutions mieux adaptées aux réalités politiques diverses que l'on a trop longtemps tenté d'intégrer dans des cadres inadaptés.

Cela dit, passons maintenant à la prochaine série de défis que le fédéralisme nous invite à confronter. L'examen de ces défis fonctionnels nous permettra de mieux mettre en relief les réels défis politiques auxquels fait face le fédéralisme, défis que nous examinerons dans la troisième et dernière partie de cet article.

\section{Quelques défis fonctionnels du fédéralisme}

L'État moderne est largement perçu comme un fournisseur de services. Si un Hobbes voyait dans l'État le fournisseur de services de sécurité au $17^{\mathrm{e}}$ siècle, la gamme de services offerts par l'État s'élargira avec le temps. En fait, Léon Duguit, au début du $20^{\mathrm{e}}$ siècle, annonçait clairement la transformation d'une conception de l'État comme " autorité publique " en une simple conception des arrangements institutionnels du "service public ${ }^{6}$. L'État démystifié et déshabillé de ses apparats métaphysiques ne serait qu'un ensemble de fournisseurs de services. Les travaux du philosophe Joseph Raz illustrent bien cette attente : l'autorité se justifie par le service qu'elle rend ${ }^{7}$. L'autorité de l'État et du droit se justifierait donc par le niveau d'efficience avec lequel ils peuvent offrir leurs services aux individus se trouvant sous leur autorité. Ainsi, selon cette conception, l'État servirait à résoudre des problèmes d'action collective en organisant la coopération, en mettant en place des structures de coordination et en assurant la survie et l'épanouissement de la société.
Vue sous cet angle réducteur et purement fonctionnel, la Fédération - tout comme l'État, d'ailleurs - ne serait qu'une société. Une association, une Gesellschaft au sens où l'entendait le sociologue Ferdinand Tönnies'. Dans cette perspective, les individus peuvent s'attendre à une efficience maximale dans la gestion et l'offre de services de l'organisation à laquelle ils contribuent financièrement en payant leurs taxes et impôts. Si le fournisseur de services n'est pas assez efficace, le consommateur (plutôt que le citoyen) cherchera légitimement à aller voir le compétiteur pour obtenir un service plus efficace et à plus bas prix. Nous ne parlons pas nécessairement ici d'autres États, mais plutôt d'autres organisations publiques ou privées qui pourraient rendre lesdits services. Si l'on a longtemps décrit l'État comme un arbre à trois branches - législative, exécutive et judiciaire -, il semble que cet arbre soit de plus en plus caché par les feuilles des contrats octroyés à des organisations parapubliques ou privées pour qu'elles accomplissent les fonctions traditionnellement attendues de l'État: pensons, entre autres, aux prisons privées, à la sécurité militaire assurée par des entreprises privées, aux tribunaux d'arbitrage de tous genres ou aux organismes volontaires de réglementation et d'harmonisation normative. L'État délègue plusieurs de ses tâches à différents fournisseurs de services pour tenter d'en réduire le coût et il crée une série d'instruments de gouvernance qui effacent la distinction entre acteurs publics et privés ${ }^{9}$. L'État - quel que soit le type de régime qui le compose - fait donc face à une concurrence venue de l'extérieur et qui a été rendue possible par son assimilation à un fournisseur de services; il doit alors prendre des mesures de manière à optimiser son efficacité au risque de perdre sa légitimité.

Dans cette perspective, le premier défi que le fédéralisme devrait relever, sur le plan fonctionnel, semble celui de démontrer qu'il est capable d'offrir des services publics de manière au moins aussi efficiente que les régimes pouvant s'y substituer. Certains souligneront le fait que la concurrence entre les diverses composantes d'une Fédération (État central et États fédérés) incite à l'expérimentation et au développement de méthodes de gouvernance plus efficaces, 
alors que d'autres feront plutôt état des risques d'une course vers les standards planchers. Toutefois, il faut se souvenir que le premier défi fonctionnel du fédéralisme ne consisterait pas en celui d'égaler ou de surpasser toute autre forme de régime au chapitre de l'efficience dans l'abstrait. Ce premier défi consisterait plutôt en l'optimisation de l'efficience du régime en fonction des réalités politiques concrètes dans lesquelles il évolue. La critique selon laquelle une Fédération serait moins efficiente qu'un autre régime en raison de l'autonomie réelle que ses diverses composantes possèdent repose sur un modèle abstrait qui ne prend pas nécessairement en compte le fait que les États ne naissent pas ex nihilo. Or, les régimes étatiques sont construits sur la réalité politique en place. Ainsi, la Fédération peut très bien être le régime le plus efficient dans les conditions concrètes où elle est réalisée. En effet, lorsque l'autre possibilité à laquelle on compare la Fédération est celle de la complète indépendance des parties qui la composent, ou encore, un État unitaire qui doit faire face à une contestation armée dans une région, il n'est pas difficile de concevoir que la Fédération puisse représenter le système le plus efficient dans les circonstances.

Cela explique le fait que si le fédéralisme est une réponse à une situation locale particulière, il est normal de s'attendre à ce que différentes formes de fédéralisme se développent en fonction des besoins locaux. Le défi est alors d'user de son imagination pour développer le meilleur amalgame de pouvoirs possible compte tenu des circonstances. La comparaison avec d'autres Fédérations sera utile pour nourrir cette imagination et pour examiner les avantages et inconvénients vécus par d'autres modèles dans leur contexte propre. Il faut toutefois être très prudent lorsque l'on cherche à tirer des généralisations relativement aux pratiques étrangères. En effet, ce n'est pas parce que le gouvernement central d'une Fédération possède un pouvoir exclusif sur une matière qu'il va de soi qu'une telle attribution serait appropriée dans une autre Fédération. Après tout, si le concept de fédéralisme nous libère du carcan conceptuel de l'État-Nation, ce n'est pas pour nous enfermer dans un nouveau. Le fédéralisme est une doctrine immensément pragmatique pouvant s'adapter aux réalités concrètes; il faut en faire usage en gardant cet élément en tête si nous voulons réellement tirer profit de sa spécificité. Bref, si le choix d'une structure fédérale reposait uniquement sur l'objectif d'optimisation de l'efficience du régime choisi dans la prestation de services publics, ce choix ne pourrait se faire quau regard d'un examen empirique des conditions politiques réelles « sur le terrain ».

Notons en terminant sur ce point que si le fédéralisme permet de démembrer les différents pouvoirs de l'État et de les distribuer à diverses institutions, cette distribution n'a pas pour but principal d'optimiser l'efficience des services publics. Ainsi, une Fédération qui prétend fonder la répartition de ses compétences entre ses divers paliers de compétence selon le principe de subsidiarité - principe voulant que les décisions soient prises le plus près possible du citoyen et que le recours à une instance supérieure n'ait lieu qu'en cas d'incapacité de l'instance de proximité de gérer la question - ne vise pas d'abord et avant tout à créer un système où on allège la tâche des gestionnaires hiérarchiquement supérieurs en donnant plus d'autonomie décisionnelle aux instances inférieures sur des enjeux de moindre importance, au contraire! Le principe d'allocation des pouvoirs qu'est celui de la subsidiarité vise plutôt à remettre les pouvoirs les plus importants pour une communauté entre ses propres mains; l'autonomie n'est pas ici un moyen, mais une fin de la répartition des pouvoirs.

Dire cela vient évidemment heurter de plein fouet la prémisse sur laquelle cette section s'est articulée jusqu'à présent, soit que le critère de justification de l'action étatique est le service que celle-ci rend. Or, si nous ne l'avions pas encore souligné parce que nous avons refusé ici d'élaborer une théorie de l'État, il importe maintenant de dire qu'il est risqué sur le plan théorique de concevoir l'État comme un simple fournisseur de services (d'ordre, de défense, réglementaire, etc.). Si cela nous permet d'imaginer que la multiplicité des ordres étatiques n'est qu'un pas dans la direction d'une ouverture plus générale à la concurrence dans la fourniture de services publics, cela 
nous fait perdre une différence qualitative importante entre l'État et les autres institutions servant simplement à accomplir des tâches fonctionnelles. En effet, il semble que l'existence de l'État soit antérieure à ses fonctions, à la différence de ces autres institutions. C'est-àdire que l'État incarne une existence commune avant même d'offrir certains services à ses membres, ce qui le distingue d'organisations qui naissent pour offrir des services à leurs clients. Penser l'État comme un simple fournisseur de services alors que l'on cherche à comprendre le fédéralisme nous ramènerait à la case de départ puisque le fédéralisme est d'abord et avant tout une solution aux identités politiques multiples plutôt qu'à des besoins d'efficience. Le choix du fédéralisme plutôt que d'un autre régime dans une situation concrète particulière, même s'il est influencé par des enjeux d'efficience, repose ultimement sur autre chose que ce dernier critère. Il repose aussi sur le fait que la Fédération permet de mieux accomplir la fonction de représentation nécessaire à la légitimation de l'État en question.

Attardons-nous maintenant à un deuxième défi fonctionnel : la pratique même du fédéralisme. Penchons-nous sur la concurrence verticale entre les composantes de la Fédération qui peut provoquer la surenchère des services visibles, et son corollaire, l'abandon des services à faible visibilité. Puisque la légitimité de l'État, selon le modèle décrit plus haut, dépend en partie des services rendus aux individus et que l'État démocratique doit faire élire ses dirigeants, il existe une concurrence entre les paliers de gouvernement pour s'approprier les domaines à haute visibilité. Il ne s'agit pas simplement pour ces paliers d'avoir juridiction sur des domaines utiles, mais aussi d'occuper des espaces visibles de gouvernance.

C'est là une des raisons pour lesquelles il est difficile d'en arriver à maintenir des structures fédérales qui respectent le principe de subsidiarité. Comme nous venons de le voir, selon le principe de subsidiarité, les décisions touchant le plus directement les personnes doivent être prises le plus près d'elles de manière à mieux respecter les besoins locaux; l'autorité générale ne devrait intervenir qu'en cas d'incapacité d'agir de la part de l'autorité particulière. Or, une telle division relègue l'autorité plus générale, l'État central, à des compétences utiles, mais moins visibles. Pour prendre un exemple canadien, il est difficile de mener des campagnesélectorales principalement sur des thèmes comme les services postaux ${ }^{10}$, le recensement et les statistiques ${ }^{11}$, les amarques, les bouées, les phares ${ }^{12}$ ou les poids et mesures ${ }^{13}$. Les thèmes militaires ${ }^{14}$ peuvent être porteurs dans certaines circonstances, mais en temps de paix, il ne s'agit pas nécessairement de thèmes majeurs. Il y a bien les thèmes économiques tels que le cours monétaire et le monnayage ${ }^{15}$, les banques, l'incorporation des banques et l'émission du papier-monnaie ${ }^{16}$, les lettres de change et les billets promissoires ${ }^{17}$ ou l'intérêt de l'argent ${ }^{18}$, mais là encore, bien qu'il s'agisse d'éléments cruciaux pour le bon fonctionnement de la société, il ne s'agit pas forcément de thèmes qui mobilisent les masses d'électeurs nécessaires au succès électoral. Dans ces circonstances, l'État fédéral sera tenté d'occuper des espaces autrement réservés aux États fédérés afin de pouvoir mettre la main sur des enjeux " électorables » : soit la santé, la culture, l'éducation; enjeux sur lesquels le citoyen moyen peut se faire une opinion assez facilement et se sentir touché dans ses aspirations. Cela peut donc avoir pour effet de créer une surenchère dans les domaines à haute visibilité de la part de l'État central et des États fédéraux et un relatif sous-investissement dans des domaines à capital politique moindre.

Cette recherche d'espaces de visibilité donne aussi parfois lieu à des contorsions constitutionnelles et administratives plutôt remarquables dans le but de justifier légalement l'action d'un palier dans le champ exclusif de l'autre. Cette question ouvre à elle seule tout un pan d'études sur le fédéralisme : quels sont les divers moyens, les divers véhicules juridiques ou parajuridiques employés pour mettre en ouvre les pouvoirs étatiques dans une Fédération? Après tout, puisque l'État n'agit pas uniquement en adoptant des lois, mais aussi en concluant des contrats, en procédant à des octrois, en taxant, etc., l'étude d'une Fédération ne peut se contenter d'examiner les seules missions législatives prévues pour chaque unité 
composante dans le texte de sa constitution; elle doit examiner l'ensemble des moyens utilisés par celles-ci pour réaliser leurs missions et, souvent, pour aller bien au-delà. Rappelons que l'État n'a pas qu'un imperium, il a aussi un dominium avec lequel il peut se livrer à des abusus... ${ }^{19}$

Le défi consiste ici à trouver la distribution appropriée des pouvoirs qui assurera non seulement une certaine efficience au tout et un espace " électorable » pour chaque palier, mais aussi une allocation qui sera respectueuse de la fonction de représentation des diverses communautés que la Fédération cherche à accomplir. Puisque ces communautés possèdent des caractéristiques variables, il est possible que la fonction de représentation requière un "fédéralisme asymétrique » où les États fédérés ne jouissent pas tous de droits, pouvoirs et obligations identiques. Toutefois, sur le plan politique, certains y voient des difficultés. Nous allons donc passer à l'examen de quelques défis politiques pour le fédéralisme.

\section{Quelques défis politiques du fédéralisme}

Si les défis discutés dans la section précédente s'inscrivaient principalement sous le registre de la société dans le sens où Tönnies l'employait, les défis présentés dans cette section relèvent plutôt du registre de la communauté, de la loyauté et de l'appartenance. C'est là, à notre avis, tout l'intérêt du fédéralisme. Si cette partie de notre article traite des enjeux politiques du fédéralisme, elle ne visera évidemment pas à traiter de l'ensemble des questions partisanes qui peuvent se poser dans le cadre d'une Fédération.

Nous discuterons plutôt d'enjeux politiques fondamentaux. Nous allons en fait aborder une question fort semblable à celle que Carl Schmitt voyait au cœur de la notion du politique ${ }^{20}$, soit la distinction ami/ennemi. Le principal défi politique du fédéralisme, nous semble-t-il, est de transcender cette distinction.

En effet, au sein de Fédérations, il n'est pas rare de constater la présence de deux types de «souverainistes » quis'opposent, ceux qui croient en la nécessaire atteinte de la souveraineté de l'État fédéré qui est le leur et ceux qui croient en la nécessaire affirmation de la souveraineté de l'État central. Ces deux groupes s'entendent pour dire qu'il ne peut ultimement $y$ avoir qu'un "Nous » qui puisse compter et que le débat doit donc porter sur qui est réellement le «Nous » et qui est l'Autre, l'ennemi, dans le sens existentiel. Bien qu'ils s'opposent les uns aux autres sur l'identité du "Nous ", ils sont en fait des alliés objectifs à l'encontre des fédéralistes qui eux prétendent qu'il est possible de faire partie du " Nous » fédéral tout en faisant partie du « Nous » fédéré. Ces deux " Nous », bien que distincts, ne pourraient être vus comme ennemis puisqu'ils sont construits sur une même matière, le citoyen ne pouvant pas se considérer être à la fois lui-même et la négation de lui-même. Le défi est donc de maintenir cette pensée fédérale et d'éviter de retomber dans la pensée souverainiste qui affaiblit la Fédération par ses forces centrifuges ou centripètes.

Pour éviter de retomber dans cette logique "souverainiste ", il est utile de mieux comprendre comment elle se déploie dans les débats politiques au sein des Fédérations. Pour ce faire, nous allons brièvement présenter une grille d'analyse du phénomène.

À la section précédente, nous suggérions qu'il fallait se garder de voir en la Fédération un simple arrangement visant principalement l'efficience. En fait, nous ajouterions maintenant qu'il faut se garder de voir la Fédération comme un simple "régime fonctionnel». Ce que nous appelons " régime fonctionnel " est une mécanique institutionnelle dont l'objectif est de résoudre des problèmes fonctionnels comme celui de coordonner des conduites. Ces institutions collectives existent pour atteindre certains buts, elles sont des moyens et non des fins. Un cas paradigmatique d'un tel régime est sans doute l'Organisation internationale de normalisation (ISO) tel qu'il est perçu par la plupart des gens qui n'y voient qu'une organisation permettant de résoudre certains problèmes de coordination. $\mathrm{Si}$ le moindre sens identitaire résulte de la participation à un régime fonctionnel, il s'agit alors d'un simple effet incident (sauf si le régime a été 
mis en place spécifiquement dans le but de produire un sentiment identitaire chez ses participants). À un autre bout du spectre des agents collectifs, nous retrouvons ce que nous nommons des "communautés existentielles». Ces dernières sont des communautés à travers lesquelles l'identité des individus est constituée par un profond sentiment d'" amour ", de loyauté, envers les autres membres du groupe. Nous n'évoquons évidemment pas ici l'amour romantique; plutôt quelque chose qui transcende la philia, mais qui n'est pas nécessairement aussi universel que l'agapè. En d'autres mots, la communauté existentielle est celle qui permet au «Soi » de transcender son sens individuel. Ces communautés, du point de vue interne du membre qui y est attaché, ne sont pas des moyens, mais plutôt des fins en soi. Un cas paradigmatique de cet être-ensemble serait sans doute le point de vue catholique selon lequel l'Église est le corpus mysticum du Christ. ${ }^{21}$ Évidemment, la question de savoir si une institution est principalement une incarnation d'une communauté existentielle ou si elle relève plutôt du régime fonctionnel peut faire l'objet de controverses très vives parmi les gens qui participent à l'institution en question.

Maintenant que nous avons présenté les concepts de base de notre grille d'analyse, voici les postulats sur lesquels cette grille repose : (1) il existe des " régimes fonctionnels " et des " communautés existentielles »; (2) les premiers fonctionnent selon une logique d'efficacité alors que les secondes évoluent dans un registre existentiel; (3) la qualification d'une institution peut faire l'objet de controverses puisque ce qui fait qu'elle relève davantage du régime fonctionnel ou de la communauté existentielle n'est pas un élément intrinsèque à l'institution elle-même, mais participe plutôt du rapport que les agents entretiennent avec elle (il est donc possible qu'une institution soit considérée comme un simple régime fonctionnel par l'un alors que l'autre y verra l'incarnation d'une communauté existentielle); et (4) une personne peut appartenir à plus d'une communauté existentielle à la fois et son attachement à chacune de ces communautés peut varier en intensité.
Cette grille nous aide à comprendre, par exemple, quelques incidents issus de la vie politique de la Fédération canadienne. Elle nous aide notamment à comprendre plusieurs malentendus entre la province de Québec et ce que les Québécois appellent souvent le « Rest of Canada » ou "R.O.C ». Bien que les ÉtatsNations se présentent sans trop de difficulté comme étant l'institutionnalisation d'une communauté existentielle, l'État multinational n'est pas toujours vu de cette manière par les individus qui le composent - à plus forte raison par les "souverainistes " cherchant à réaliser l'indépendance de leur État fédéré. En gardant cela en tête, on peut mieux comprendre le commentaire très controversé d'un ancien premier ministre souverainiste québécois, Lucien Bouchard, à l'effet que le "Canada est divisible puisqu'il ne s'agit pas d'un vrai pays $»^{22}$, et la furie qu'il a provoquée chez les " souverainistes canadiens " en 1996. On peut interpréter l'affirmation de $\mathrm{M}$. Bouchard au sens où le Canada ne serait pas une communauté existentielle, mais un simple régime fonctionnel, et partant, qu'il pourrait être fragmenté à volonté par les communautés existentielles pour qui ce régime a été mis en place. Pour les nationalistes canadiens - fédéralistes ou " souverainistes canadiens "-, ceci était non seulement erroné sur le plan descriptif, mais également une insulte à leur sens identitaire parce que cela niait que leur existence eût quelque substance. Une situation analogue se produit lorsqu'un anglophone ne comprend pas pourquoi les francophones du Québec réclament des mesures particulières pour protéger leur langue plutôt que de simplement laisser le " marché linguistique » s'en charger. La langue, pour bien des francophones du Québec, n'est pas qu'un instrument de communication, il s'agit d'un élément qui est constitutif du soi transcendant.

Ainsi, plusieurs controverses politiques découlent inconsciemment de désaccords quant à savoir si une institution relève principalement d'un caractère existentiel ou plutôt d'un caractère fonctionnel. De cette qualification découle toute une logique, toute une série de critères évaluatifs qui ne sont pas compatibles les uns avec les autres et qui alimentent la controverse. C'est 
ici que le caractère moniste du souverainisme vient créer d'importantes tensions politiques, car ce cadre conceptuel laisse bien peu de place pour concevoir l'appartenance à plus d'une communauté politique comme le fédéralisme tente de le rendre possible. "Le corps politique ne peut avoir deux têtes sans être un monstre", diront les souverainistes. La double loyauté est alors pratiquement inconcevable.

Cette façon de voir les choses entraîne d'importantes conséquences pour la psychologie politique des citoyens. Non seulement doiventils choisir leur camp entre la communauté politique incarnée par l'État fédéré et celle de l'État central, mais les enjeux des affrontements politiques entre les paliers de gouvernement sont très importants : perdre signifie perdre son identité, perdre une possibilité de survivre à sa propre mort grâce à la survie de la communauté qui transcende l'individu. Cela devient donc un jeu à somme nulle. Tout compromis devient " trahison " et l'entreprise politique prend alors la forme d'une "lutte » ou d'une " guerre idéologique ». La coopération entre les paliers gouvernementaux et leur égale coexistence deviennent difficilement intelligibles lorsque cette perspective souverainiste domine.

Or, lorsque cette conception " souverainiste " s'empare des politiciens contrôlant l'État central, paradoxalement, cela mène à des stratégies visant à maintenir l'unité de la Fédération qui sont complètement contre-productives. En effet, en " combattant» les tentatives de légitimation des États fédérés, ils ne réussissent qu'à aliéner les gens qu'ils tentent de rallier à leur camp. Celui qui siège au centre de l'État ferait bien d'écouter la sagesse de l'adage woloff suivant lorsqu'il tente de lutter contre les forces souverainistes centrifuges des territoires excentrés : "Lu la mar mayul, màtt du la komay. » ("Ce que lécher ne peut pas donner, mordre ne le donne pas. ") La conception " souverainiste " de l'État central tend en effet à oublier que l'attachement à une communauté politique est d'abord et avant tout fait d'émotion et d'intériorisation et que ces dispositions, tout comme l'amour, ne peuvent être imposées par la force. "Aime-moi, sinon! » n’a jamais été le meilleur moyen de gagner le cœur d'autrui... Le principal défi politique du fédéralisme - mais aussi, son principal avantage comparativement à d'autres régimes politiques - est donc qu'il peut et qu'il doit développer des moyens d'accommoder les différentes appartenances aux diverses communautés politiques unies pour former une entité étatique.

C'est ce sens d'appartenance à la communauté existentielle incarnée par l'État qui donne sa légitimité première à un gouvernement. Le défi consistant en la réconciliation de la "diversité et de l'unité " n'est pas un simple slogan vide. Ce qui est en jeu est que l'idée de l'autonomie gouvernementale - le self-government - exige que le Soi gouverne (donc, que la communauté existentielle soit la source des lois). Parallèlement, l'idée même de gouvernement est associée à l'idée qu'il faut efficacement prendre des décisions collectives et les mettre en œuvre de manière efficiente; l'État est aussi une forme de régime fonctionnel. Le fédéralisme est donc une tentative de marier harmonieusement les attentes de diverses communautés existentielles et leurs besoins fonctionnels respectifs.

Cela explique, entre autres, pourquoi le fédéralisme asymétrique peut s'avérer une solution tout à fait acceptable selon les conditions politiques particulières d'une Fédération. C'est notamment le cas lorsqu'un État fédéré est majoritairement peuplé par un groupe formant une communauté existentielle qui est autrement une minorité nationale au sein de la Fédération. Le fédéralisme asymétrique est donc une solution pratique qui permet dans certains cas de prendre en compte la diversité des appartenances politiques - et leur intensité respective - au sein de la Fédération.

\section{Conclusion}

Concluons en soulignant que la reconnaissance des demandes d'autonomie de régions ou de groupes distincts ne devrait pas être vue comme une menace à l'unité de l'État, mais plutôt comme le signe qu'une Fédération ou un État empruntant des formes fédérales est capable d'accommoder plusieurs sens de l'attachement au sein même de son appareil institutionnel. 
En d'autres mots, cela devrait être vu comme la preuve que l'organisation étatique est suffisamment flexible pour accueillir une multitude de communautés existentielles tissées les unes aux autres. Cela devrait être un signe de réussite et non d'échec. On dit souvent que la politique est l'art du possible; nous ajoutons que le fédéralisme, lui, se veut l'imagination au service du possible.

\section{Notes}

* Hugo Cyr, LL.B., B.C.L. (McGill), LL.M. (Yale), LL.D. (U. de Montréal) est professeur de droit public et de théorie du droit à la Faculté de science politique et de droit, Université du Québec à Montréal et membre du Barreau du Québec. Le présent article est une version légèrement remaniée du texte de l'allocution que l'auteur a livrée à Dakar (Sénégal), le 5 décembre 2011, lors de la « Journée de réflexion sur la diversité des démocraties » organisée par le "Groupe des amis de la francophonie » composé des ambassadeurs des États membres de l' " Organisation internationale de la Francophonie » (OIF) et d'autres organisations francophones de Dakar.

1 Le grand publiciste Ivor Jennings écrivait non sans rappeler implicitement la fameuse formule de Churchill sur la démocratie - qu'une " "Federation" is not a magical formula. It is nothing more than the name of a complicated system of government which nobody would wish to see established anywhere if he could think of a better. » (Ivor Jennings, A Federation for Western Europe (Cambridge, Cambridge University Press, 1940, p 3)).

2 C'est notamment le cas des règles du droit international coutumier.

3 Loi constitutionnelle de 1867 (R-U), 30 \& 31 Vict, c 3, art 55, reproduite dans LRC 1985, ann II, nº 5 [Loi constitutionnelle de 1867].

4 Le temps ne me permet pas ici d'explorer plus à fond les tensions entre le concept de souveraineté et le fédéralisme, mais celles et ceux qui seraient intéressés à lire un peu plus sur cette question sont invités à consulter mon article $\mathrm{H} \mathrm{Cyr,}$ "L'inadéquation du concept de souveraineté à la réalité fédérale » dans A-G Gagnon et $\mathrm{F}$ Requejo, éds, Nations en quête de reconnaissance: Regards croisés Québec-Catalogne, Bruxelles, Presses interuniversitaires européennes/ Peter Lang, 2011, 227.

5 Voir notamment Antoine Messara, «Principe de territorialité et principe de personnalité en fédéralisme comparé : Le cas du Liban et perspectives actuelles pour la gestion $\mathrm{du}$ pluralisme » dans Jean-François GaudreaultDesBiens et Fabien Gélinas, éds, Le fédéralisme dans tous ses états : gouvernance, identité et méthodologie, Cowansville (Qc), Yvon Blais, 2005, 227.

6 Voir notamment Léon Duguit, Les transformations du droit public, Paris, Armand Collin, 1913, ch 2.

7 Voir notamment son ouvrage classique Joseph Raz, The Authority of Law: Essays on Law and Morality, Oxford, Oxford University Press, 1983.

$8 \quad$ Ferdinand Tönnies, Communauté et société. Catégories fondamentales de la sociologie pure, traduit par J Leif, Paris, Presses universitaires de France, 1944.

9 Pensons, par exemple, à ces «Quasi-Autonomous Non-Governmental Organisations » (ou "QUANGOs») que l'on retrouve dans le monde anglo-saxon où il s'agit d'entités privées auxquelles l'État a dévolu certaines fonctions étatiques telles que la réglementation d'un secteur d'activité ou la fourniture de certains services aux usagers. Ou encore, pensons aux «partenariats publics-privés » («PPP ») caractérisés par un appel par l'autorité publique à des partenaires privés pour le financement, la construction ou la gestion de biens servant à la fourniture de services publics.

10 Loi constitutionnelle de 1867 , supra note 3 , art 91(5).

11 Ibid, art 91(6).

12 Ibid, art. 91(9).

13 Ibid, art. 91(17).

14 Ibid, art. 91(7).

15 Ibid, art. 91(14).

16 Ibid, art. 91(15).

17 Ibid, art. 91(18).

18 Ibid, art. 91(19).

19 Au Canada, par exemple, le palier fédéral utilise son pouvoir de taxation pour amasser des sommes qui dépassent ses besoins découlant de ses propres champs de compétence et il retourne ensuite une partie de ces sommes aux provinces pourvu que celles-ci mettent en place certains régimes de prestation de service conformes à ses exigences. Les fondements constitutionnels de cette pratique datant de plus de cinquante ans sont toutefois fort controversés.

20 Carl Schmitt, «La notion du politique » dans La notion du politque; Théorie du partisan, traduit par Marie-Louise Steinhauser, Paris, Flammarion, 1992, 57. Voir H Cyr, « Fédéralisme 
et le concept du politique chez Carl Schmitt : Le problème de l'homogénéité de l'unité politique et du pluralisme fédéral » dans M Seymour et $\mathrm{G}$ Laforest, éds, Le fédéralisme multinational en perspective : un modèle viable?, Bruxelles, Peter Lang, 2011, 37.

21 Voir Ernst H Kantorowicz, The Kings Two Bodies: A Study in Mediaeval Political Theology, Princeton, Princeton University Press, 1957.

22 Philip Authier, "Bouchard says no to partition " The [Montreal] Gazette (28 January 1996) c 12. 